\title{
Scaling of Near-Wall Flows in Quasi-Two-Dimensional Turbulent Channels
}

\author{
D. Samanta, ${ }^{2}$ F. Ingremeau, ${ }^{1}$ R. Cerbus, ${ }^{2,3}$ T. Tran, ${ }^{4}$ W. I. Goldburg, ${ }^{3}$ P. Chakraborty, ${ }^{2}$ and H. Kellay ${ }^{1}$ \\ ${ }^{1}$ University of Bordeaux, LOMA (UMR 5798 CNRS), 351 cours de la Libération, 33405 Talence, France \\ ${ }^{2}$ Okinawa Institute of Science and Technology, Okinawa, Japan 904-0495 \\ ${ }^{3}$ Department of Physics and Astronomy, University of Pittsburgh, Pittsburgh, Pennsylvania 15260, USA \\ ${ }^{4}$ Division of Thermal and Fluids Engineering, School of Mechanical and Aerospace Engineering, \\ Nanyang Technological University, Singapore 639798, Singapore
}

(Received 13 February 2014; published 9 July 2014)

\begin{abstract}
The law of the wall and the log law rule the near-wall mean velocity profile of three-dimensional turbulent flows. These well-known laws, which are validated by legions of experiments and simulations, may be universal. Here, using a soap-film channel, we report the first experimental test of these laws in quasi-two-dimensional turbulent channel flows under two disparate turbulent spectra. We find that despite the differences with three-dimensional flows, the laws prevail, albeit with notable distinctions: the two parameters of the log law are markedly distinct from their three-dimensional counterpart; further, one parameter (the von Kármán constant) is independent of the spectrum whereas the other (the offset of the $\log$ law) depends on the spectrum. Our results suggest that the classical theory of scaling in wall-bounded turbulence is incomplete wherein a key missing element is the link with the turbulent spectrum.
\end{abstract}

DOI: 10.1103/PhysRevLett.113.024504

PACS numbers: 47.27.nd

Turbulent flows over walls are ubiquitous, whether in natural settings (e.g., rivers, atmospheric boundary layers) or in industrial ones (e.g., channels, pipes) [1,2]. The wall shear stress-which sets the cost of pumping liquid in a pipe, for example-slows the mean flow near the wall, bringing the flow at the wall to zero velocity (the no-slip boundary condition). For three-dimensional wall-bounded turbulent flows, Prandtl, in 1925, postulated the dominant role of wall shear stress and fluid viscosity to derive the law of the wall for the scaling of the mean velocity profile (MVP) near the wall [2]. The near-wall spatial domain, where the law of the wall reigns, can be divided into three layers. Starting from zero velocity at the wall, the mean velocity linearly increases with distance from the wall in the viscous sublayer, then flexes in the buffer layer to blend into a logarithmic variation with further distance from the wall in the log layer. This logarithmic variation, the log law, was derived by von Kármán in 1930 and is widely considered to be a cardinal result in the study of 3D turbulent flows.

Mathematically, the law of the wall for the MVP, $\langle U(y)\rangle$ [where $U(y)$ is the streamwise velocity at a distance $y$ from the wall, and \langle\rangle denotes averaging over time] is typically cast in terms of the so-called wall variables, $U^{+}=$ $(\langle U(y)\rangle) /\left(\sqrt{\tau_{w} / \rho}\right)$ and $y^{+}=y /\left(\nu \sqrt{\rho / \tau_{w}}\right)$, where $\tau_{w}$ is the wall shear stress, and $\rho$ and $\nu$, respectively, are the density and the kinematic viscosity of the fluid. This law reads: $U^{+}=f\left(y^{+}\right)$, where the functional form of $f$ remains unspecified. In the viscous sublayer, $U^{+}=y^{+}$. In the log layer, the $\log$ law reads: $U^{+}=(1 / \kappa) \ln \left(y^{+}\right)+B$, where $\kappa$ is known as the von Kármán constant and $B$ as the offset of the $\log$ law. At high Reynolds numbers (Re), the log region spans most of the spatial domain of the law of the wall, and, as Prandtl demonstrated, for a given Re the log law can be used to predict the wall shear stress [2]. To that end, precise measurements of the two parameters, $\kappa$ and $B$, are crucial in estimating the wall shear stress. A multitude of studies have shown that at high Re these parameters are independent of Re, and that among different types of flows, variation in $\kappa$ is within $10 \%$ and variation in $B$ (for flows over smooth walls) within $25 \%$ [2-4]. A recent reanalysis demonstrates that the variation in these parameters among different flow types is even smaller [5]. These observations indicate that the $\log$ law may be a universal feature of 3D wall-bounded turbulent flows [6].

The classical derivation of the law of the wall and the log law is predicated on dimensional analysis and similarity arguments [2]. As such, these laws are independent of the dimension of the flow and the attendant turbulent spectrum. If there be a dependence on the dimension or the spectrum, this result would be outside the purview of the classical theory, thereby rendering the classical theory incomplete. While 3D flows allow only one turbulent spectrum (the energy cascade), 2D turbulent flows allow two disparate turbulent spectra (the enstrophy cascade and the inverse energy cascade) [8-10]. Thus, considering 2D flows permits testing the dependence of the MVP scaling laws on both the dimensionality and the turbulent spectrum. In contrast to $3 \mathrm{D}$, there is no experimental study of the MVP in 2D or quasi-2D wall-bounded turbulent flows. Further, only a few theoretical and numerical studies $[11,12]$ have addressed this problem. Here we experimentally study the near-wall MVP of quasi-2D turbulent channel flows, which we realize using a soap-film channel.

In a standard soap-film channel, the soap film, supported between two smooth nylon wires, flows down vertically due to gravity [13-17]. The flow is rendered turbulent by 
inserting a comb across the width of the channel and normal to the plane of the film. The turbulent flow thus produced exhibits features expected for decaying 2D turbulence [9], notably an enstrophy cascade, typically spanning a range of length scales between 0.1 and $1 \mathrm{~cm}$. The properties of such flows have been the subject of numerous studies, and modifications have been proposed to obtain not only an enstrophy cascade but also a coexisting inverse energy cascade at large scales and (forward) enstrophy cascade at small scales [13-18].

Recently, we introduced yet another way to generate turbulence in a soap-film channel, wherein, as illustrated in
Fig. 1(a), we replace the nylon wires with one rough wall and one smooth wall $[13,19]$. We adopt two configurations: we insert at the channel entrance, normal to the plane of the film, either a rod or a comb. Along with the forcing due to the rough wall, the former configuration renders the flow turbulent under an inverse energy cascade [Fig. 1(b)], while the latter under an enstrophy cascade [Fig. 1(c)], where both cascades typically span a range of length scales between 0.1 and $1 \mathrm{~cm}$. In previous work $[13,20]$ this empirical finding allowed us to carry out quantitative tests of the frictional drag in quasi-2D turbulent channels for the different turbulent spectra. Here we use this channel to
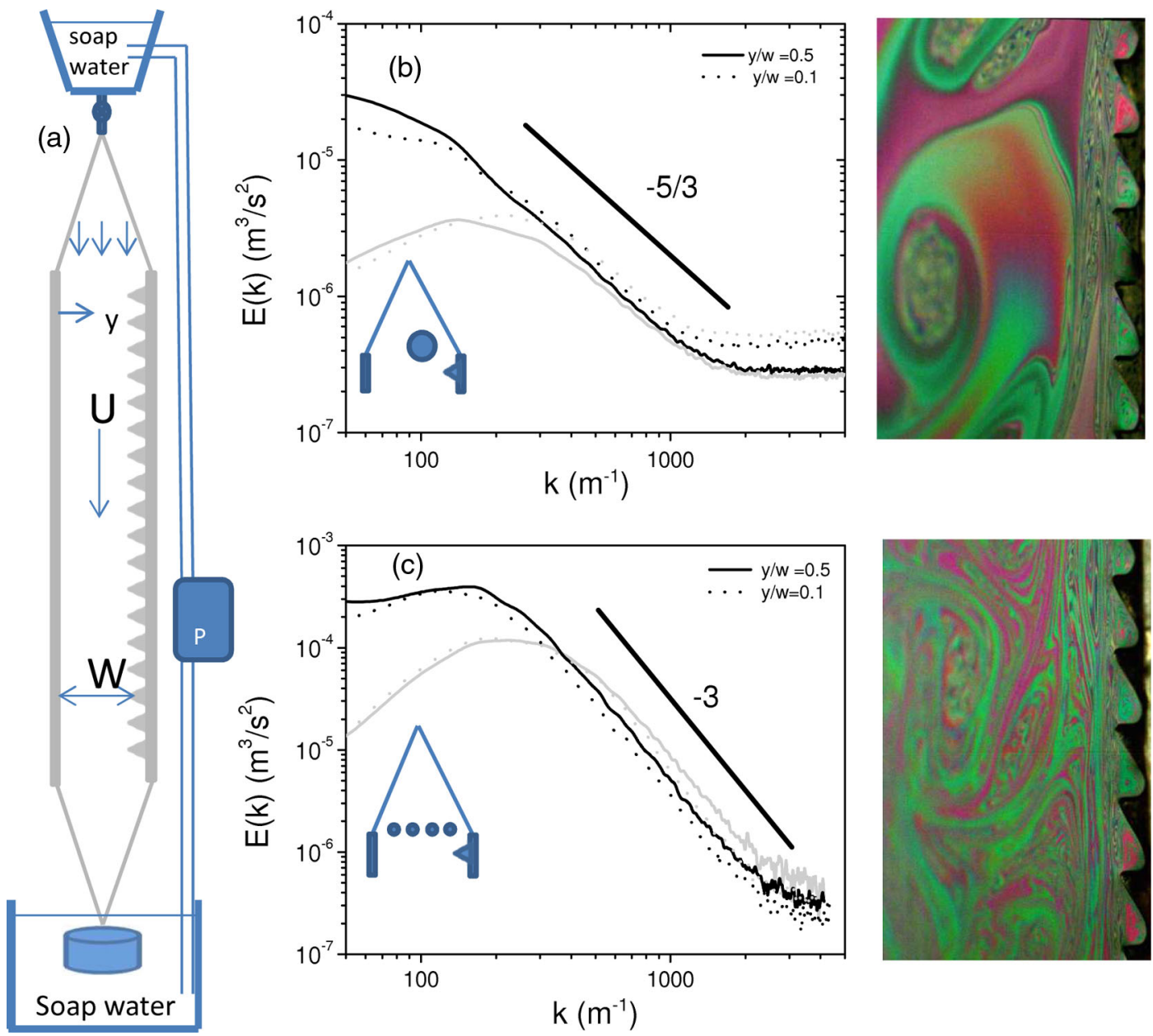

FIG. 1 (color online). Soap-film channel. (a) Schematic of the channel. Soap water (2\% dish detergent in water) from the upper reservoir feeds the soap film that is supported between a smooth wall and a rough wall. (The walls are steel blades.) After draining at the bottom, the pump $P$ brings the soap water from the lower reservoir to the upper one, thereby establishing steady flow in the channel. The injection of turbulence is controlled in two ways. (b) Experimental setup with a rod at the channel entrance. The resultant energy spectra $E(k)$ corresponds to the inverse energy cascade (where the spectral exponent is $-5 / 3$ ). The spectral exponent is the same for both the longitudinal (black) and transverse (gray) components of $E(k)$, and the same across the width of the channel (see dotted and solid lines). We compute the spectra from velocity fluctuations measured using a LDV probe sampling at about $5 \mathrm{kHz}$ acquisition rate and use Taylor's hypothesis to transform from frequency space to wave number space (see Methods section in Ref. [20]). In the adjacent photograph we show interference fringes (in white light) close to the rough wall; a large eddy shed from rod interacts with the rough wall. (c) Experimental setup with a comb at the channel entrance. The resultant energy spectra $E(k)$ corresponds to the enstrophy cascade (where the spectral exponent is -3 ). In measuring and plotting $E(k)$, we adopt the same convention as described in (b). In the adjacent photograph we show interference fringes (in white light) close to the rough wall; eddies of different scales are shed from the comb. 


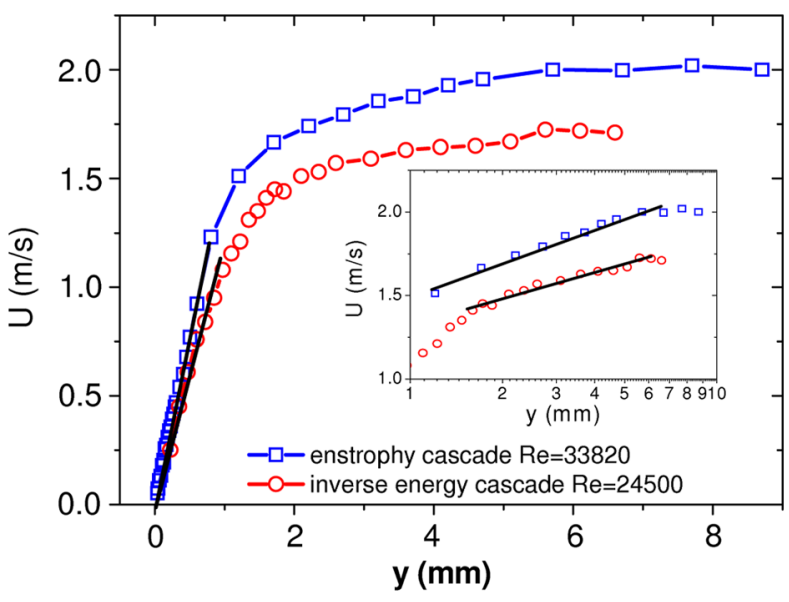

FIG. 2 (color online). Representative MVPs for both turbulent spectra. The MVPs are measured using LDV. The MVP is linear near the wall (see black lines). The inset (semilog coordinates) shows a zoomed view of the log layer (the logarithmic variation is marked by black lines).

measure the MVP in quasi-2D turbulent flows with different turbulent spectra.

Using laser Doppler velocimetry (LDV), we measure a time series of the instantaneous streamwise velocity $U(y)$ at different locations $y$ from the smooth wall. At each location, averaging $U(y)$ over time yields the MVP, $\langle U(y)\rangle$. The Reynolds number of the flow is defined using the mean velocity in the channel $\bar{U}$ [the average value of $\langle U(y)\rangle$ over $y]$ as $\operatorname{Re}=(\bar{U} W) / \nu$, where $W$ is the channel width. By varying $\bar{U}$ (through changing the inlet flow flux or the streamwise position) and by varying $W$, Re in our experiments spans the range 6000 to 90000 .

In Fig. 2 we show representative MVPs corresponding to the two turbulent spectra. In both MVPs we note features that are reminiscent of their counterparts in 3D wall-bounded turbulent flows. A linear region-the viscous sublayer-prevails near the wall, giving a well-defined shear rate; thus, the wall shear stress is $\tau_{w}=\rho \nu(d\langle U(y)\rangle) /\left.(d y)\right|_{y=0}$. (In previous work [13,20], we measured $\tau_{w}$ using this approach.) Farther away the profile flexes in the buffer layer to blend into a logarithmic variation in the log layer (see inset of Fig. 2).

To test the validity of the law of the wall and the log law, we gather MVPs for different Re and recast them in wall coordinates, $U^{+}$versus $y^{+}$. In Fig. 3 we plot the MVPs for both turbulent spectra in semilog coordinates. For each spectra, the MVPs corresponding to different Re collapse onto a universal curve in the near-wall region, thus validating the law of the wall, $U^{+}=f\left(y^{+}\right)$. (Farther away the MVPs for different Re peel away from the universal curve, entering the domain where the law of the wall no longer holds.) Examining this universal curve, we note that in the viscous sublayer, $U^{+}=y^{+}$, in the buffer layer the curve flexes toward the logarithmic region, and in the log layer (which in semilog coordinates manifests as a straight line), $U^{+}=(1 / \kappa) \ln \left(y^{+}\right)+B$, thus validating the log law [21]. At first glance our results appear to suggest an intriguing prospect: that the law of the wall and the log law are not only universal features of 3D wall-bounded turbulent flows, but also of quasi-2D wall-bounded turbulent flows. There are, however, two telling details-the parameters of the log law-to which we turn next.

First, consider the von Kármán constant $\kappa$. To compute $\kappa$ we take each MVP (such as the ones shown in Fig. 3) and replot as $y^{+}\left(d U^{+}\right) /\left(d y^{+}\right)$versu $y^{+}$, wherein we look for a plateau, which, the log law tells us, corresponds to $1 / \kappa$. For each spectra we plot the $\kappa$ thus obtained versus Re in Fig. 4. For flows with an inverse energy cascade, we find that, for the whole span of $\operatorname{Re}, \kappa$ is approximately constant, with a
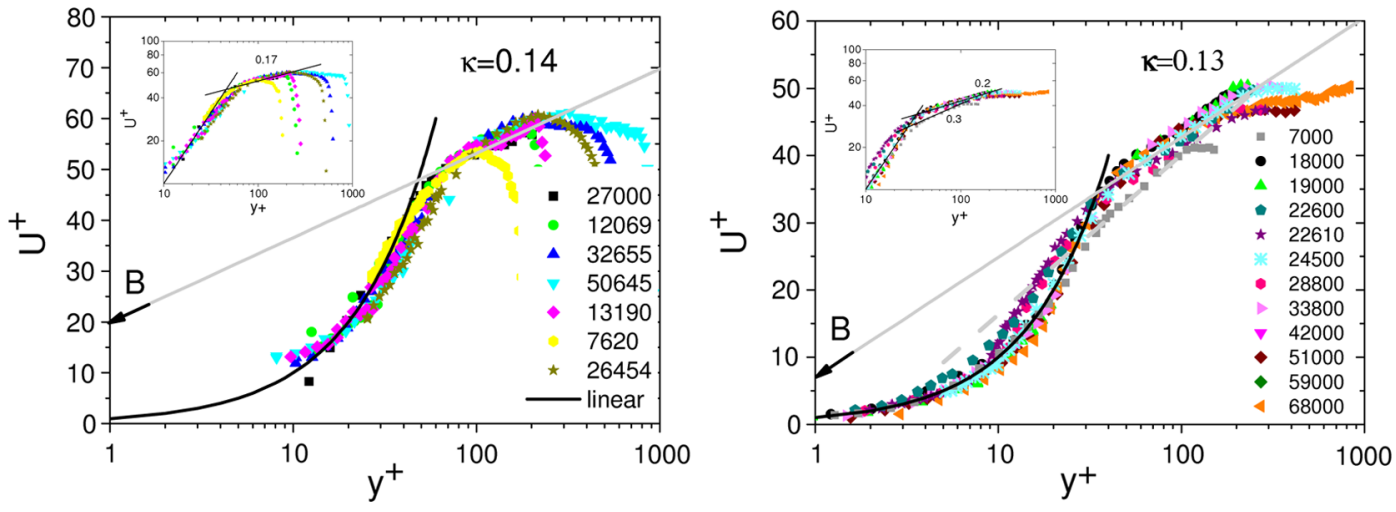

FIG. 3 (color online). MVPs as $U^{+}$versus $y^{+}$in semilog coordinates for both turbulent spectra (left, inverse energy cascade; right, enstrophy cascade) and for a range of Re. The solid black lines correspond to the viscous sublayer $U^{+}=y^{+}$. The solid gray lines, which correspond to the $\log$ law, are the best fit to the log layer with $\kappa$ as 0.14 for the inverse energy cascade and 0.13 for the enstrophy cascade (see Fig. 4). The gray dashed line in the enstrophy cascade profiles is a fit to the low Re profile with a value of $\kappa=0.09$. The intercept of these lines at $y^{+}=1$ (see arrow) yields an estimate of the value of $B$, which is $\approx 20$ for the inverse energy cascade and $\approx 7$ for the enstrophy cascade. Tests of power-law behavior are shown in the insets (log-log coordinates), where the line next to the wall corresponds to the viscous sublayer and the line farther away is the power-law region. 

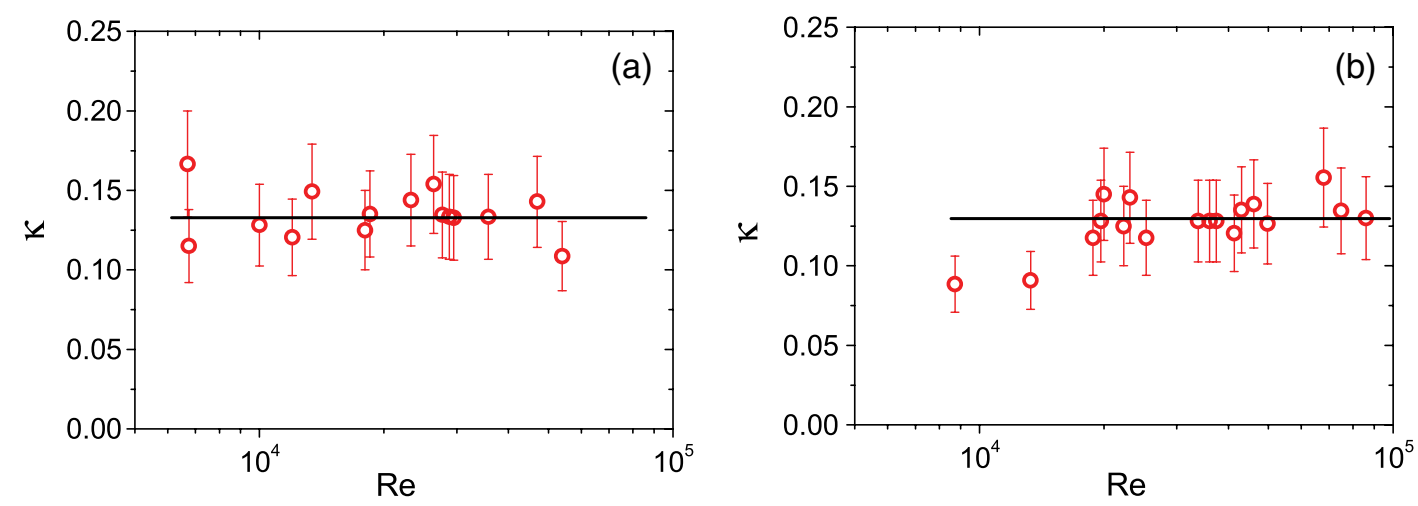

FIG. 4 (color online). Re dependence of $\kappa$, for the inverse energy cascade (a) and the enstrophy cascade (b), over the full measured range of Re. $\kappa$ is computed from the plateau region of the MVP plotted as $y^{+}\left(d U^{+}\right) /\left(d y^{+}\right)$versus $y^{+}$; the error bars represent the scatter around the plateau. The mean value (averaged over Re) of $\kappa$, shown with black lines, for the inverse energy cascade is 0.14 and for the enstrophy cascade (for $\operatorname{Re} \gtrsim 20000$ ) is 0.13 .

mean value of $\approx 0.14$. For flows with an enstrophy cascade, we find that at large $\mathrm{Re}, \kappa$ is approximately constant, with a mean value of $\approx 0.13$, and that at lower $\operatorname{Re}(\operatorname{Re} \lesssim 20000), \kappa$ assumes smaller values [22]. Within experimental uncertainty we conclude that at high $\operatorname{Re} \kappa$ in quasi-2D flows is independent of the turbulent spectra. This value of $\kappa$ is markedly different from that in 3D flows, where, to our knowledge, such small values of $\kappa$ have not been observed [23] - the reported values hover around 0.4 with a dispersion of roughly $10 \%$ [2-4].

Next, consider the offset of the log law, $B$. Limited by experimental scatter, we estimate $B$ from the MVPs shown in Fig. 3-using our above-mentioned estimate of $\kappa$, we compute a best fit line for the log law, whose intercept at $y^{+}=1$ corresponds to the value of $B$ (see the arrows in Fig. 3). (For the best fit we use all MVPs for the inverse energy cascade and MVPs at large Re for the enstrophy cascade where $\kappa$ is independent of Re.) In contrast to $\kappa, B$ depends on the turbulent spectra: for flows with an inverse energy cascade, $B \approx 20$; for flows with an enstrophy cascade, $B \approx 7$. Interestingly, this latter value is close to $3 \mathrm{D}$ channel flows where $B \approx 5$ [2].

As an aside, we note that theoretical work by Barenblatt and co-workers [26] has disputed the existence of a log law for 3D flows and proposed a power law scaling instead, where the exponent of the power law depends on Re. (This proposal also violates the law of the wall.) To test for a possible power-law MVP, we plot our data in log-log coordinates in the insets of Fig. 3. For intermediate distances from the wall, insofar as a power-law exponent can be discerned, this exponent is $\approx 0.17$ for the inverse energy cascade and $\approx 0.2$ for the enstrophy cascade except for lower Re where the exponent seems to be higher $(\approx 0.3)$. One proposal for the expected power-law exponents in the inverse energy cascade and the enstrophy cascade, respectively, are $1 / 7$ and $1 / 3$ [12]. This prediction is difficult to verify from our results.
In our considerations thus far we have treated soap-film channels as quasi-2D counterparts of 3D channels. This approach neglects two factors: the frictional drag between the falling film and surrounding air, and the Marangoni stresses due to soap molecules [27-29]. How these factors affect the near-wall MVPs in soap-film channels remains an open question.

In summary, using quasi-2D turbulent flows in soap-film channels we have verified that-the differences with 3D wall-bounded flows in dimensionality, turbulent spectra, and additional forcing notwithstanding - the near-wall MVP comprises a viscous sublayer, buffer layer, and log layer, and obeys the law of the wall and the log law. The parameters of the log law manifest notable features: the von Kármán constant $\kappa$ is independent of the turbulent spectra and is about one-third of its 3D counterpart; the offset of the $\log$ law $B$ depends on the turbulent spectra and for the enstrophy cascade is close to its $3 \mathrm{D}$ counterpart. Our results, which render the classical approach incomplete, await theoretical elucidation.

D. S., R. C., and P.C. acknowledge support from the Okinawa Institute of Science and Technology Graduate University. H. K. acknowledges support from the IUF.

[1] H. Schlichting and K. Gersten, Boundary-Layer Theory (Springer, New York, 2000).

[2] S. B. Pope, Turbulent Flows (Cambridge University Press, Cambridge, England, 2000).

[3] I. Marusic, B. J. McKeon, P. A. Monkewitz, H. M. Nagib, A. J. Smits, and K. R. Sreenivasan, Phys. Fluids 22, 065103 (2010).

[4] A. J. Smits, B. J. Mckeon, and I. Marusic, Annu. Rev. Fluid Mech. 43, 353 (2011)

[5] I. Marusic, J. P. Monty, M. Hultmark, and A. J. Smits, J. Fluid Mech. 716, R3 (2013). 
[6] While the reasons underlying this potential universality are ill understood, one possibility is furnished by a recent theory that links the MVP (a macroscopic property) with the turbulent spectrum (a statistical property of fluctuations) and identifies the log region with the inertial range of the spectrum [7]. Herein the universality of the inertial range of the turbulent spectrum dictates the universality of the log law.

[7] G. Gioia, N. Guttenberg, N. Goldenfeld, and P. Chakraborty, Phys. Rev. Lett. 105, 184501 (2010).

[8] R. Kraichnan, Phys. Fluids 10, 1417 (1967).

[9] G. K. Batchelor, Phys. Fluids 12, II-233 (1969).

[10] G. Boffetta and R. E. Ecke, Annu. Rev. Fluid Mech. 44, 427 (2012)

[11] V. S. Lvov, I. Procaccia, and O. Rudenko, Phys. Rev. E 79, 045304(R) (2009).

[12] N. Guttenberg and N. Goldenfeld, Phys. Rev. E 79, 065306 (R) (2009).

[13] H. Kellay, T. Tran, W. Goldburg, N. Goldenfeld, G. Gioia, and P. Chakraborty, Phys. Rev. Lett. 109, 254502 (2012).

[14] H. Kellay, X.-1. Wu, and W. I. Goldburg, Phys. Rev. Lett. 74, 3975 (1995).

[15] C. H. Bruneau and H. Kellay, Phys. Rev. E 71, 046305 (2005).

[16] M. A. Rutgers, Phys. Rev. Lett. 81, 2244 (1998).

[17] H. Kellay and W. I. Goldburg, Rep. Prog. Phys. 65, 845 (2002).

[18] P. Vorobieff, M. Rivera, and R. E. Ecke, Phys. Fluids 11, 2167 (1999).

[19] R. T. Cerbus and W. I. Goldburg, Phys. Fluids 25, 105111 (2013).
[20] T. Tran, P. Chakraborty, N. Guttenberg, A. Prescott, H. Kellay, W. Goldburg, N. Goldenfeld, and G. Gioia, Nat. Phys. 6, 438 (2010).

[21] In contrast to both the 3D energy cascade and the 2D inverse energy cascade, the large and small scales of turbulent velocity fluctuations, or "eddies," are statistically dependent in the enstrophy cascade (i.e., eddy interactions in Fourier space are nonlocal; see, e.g., Ref. [10]). Our finding that the log law prevails in the enstrophy cascade suggests that any general theory of the log law is rendered incomplete if it is predicated on the statistical independence of eddy interactions across the scales.

[22] Recent numerical simulations [11] of 2D channels with an enstrophy cascade have put forth such a Re dependence for $\kappa$, but their values of $\kappa$, which range from 0.2 to 0.3 , are larger than the values we find here; further, they find that $\kappa$ depends on Re for all Re. Note that Re-dependent $\kappa$ violates the law of the wall. Our results thus indicate that the law of the wall holds for flows with an enstrophy cascade when Re $\gtrsim 20000$.

[23] In 3D flows over permeable beds, $\kappa \approx 0.3$ has been observed in experiments and simulations $[24,25]$.

[24] W. P. Breugem, B. J. Boersma, and R. E. Uittenbogaard, J. Fluid Mech. 562, 35 (2006).

[25] K. Suga, Y. Matsumura, Y. Ashitaka, S. Tominaga, and M. Kaneda, Int. J. Heat Fluid Flow 31, 974 (2010).

[26] G. I. Barenblatt, A. J. Chorin, and V. M. Prostokishin, Proc. Natl. Acad. Sci. U.S.A. 94, 773 (1997).

[27] J. M. Chomaz, J. Fluid Mech. 442, 387 (2001).

[28] T. Tran, P. Chakraborty, G. Gioia, S. Steers, and W. Goldburg, Phys. Rev. Lett. 103, 104501 (2009).

[29] P. Chakraborty, T. Tran, and G. Gioia, J. Elast. 104, 105 (2011). 